\title{
Defeito ósseo de Stafne e a importância da tomografia no diagnóstico: um relato de caso
}

\section{Stafne's bone defect and importance of tomography in diagnosis: a case report}

\author{
Nathália Carvalho Silva* \\ Cássia Luana Silva Queiroz** \\ Ricardo Pitanga das Virgens ${ }^{* * *}$ \\ Jener Gonçalves de Farias ${ }^{* * * *}$ \\ Juliana Andrade Cardoso ${ }^{* * * *}$ \\ Cinthia Coelho Simões
}

\section{Resumo}

Objetivo: relatar o caso de um paciente idoso portador de defeito ósseo de Stafne. Relato de caso: paciente do sexo masculino, 74 anos de idade, foi atendido na Clínica de Odontologia da União Metropolitana de Educação e Cultura, e, após realização de radiografia panorâmica, foi evidenciada uma imagem sugestiva do defeito, sendo o paciente orientado a realizar a tomografia computadorizada fan beam para a confirmação diagnóstica. Considerações finais: o defeito ósseo de Stafne é definido como uma depressão lingual na mandíbula, de forma arredondada ou ovoide, que varia de 1 a $3 \mathrm{~cm}$ em diâmetro, com margens ósseas muito densas e bem definidas. Apresenta-se, radiograficamente, como uma imagem radiolúcida, circunscrita e unilocular na região posterior da mandíbula, abaixo do canal mandibular. É geralmente detectado em exames radiográficos de rotina, como a radiografia panorâmica, e a confirmação do diagnóstico geralmente é obtida pela realização da tomografia computadorizada fan beam ou de cone beam, por conta da obtenção de imagens que possibilitam a avaliação tridimensional, descartando, assim, outros tipos de lesões. Por se tratar de uma variação anatômica, a conduta é proservar o caso.

Palavras-chave: Cistos ósseos. Diagnóstico. Mandíbula. Tomografia.

\section{Introdução}

O defeito ósseo de Stafne (DOS) é definido como uma depressão lingual na mandíbula, de forma arredondada ou ovoide, que varia de 1 a $3 \mathrm{~cm}$ em diâmetro, com margens ósseas muito densas e bem definidas. ${ }^{1,2}$

Clinicamente foi descrito pela primeira vez por Edward Stafne, em 1942, ao realizar um estudo com 34 pacientes que apresentavam 35 lesões ósseas em mandíbula. Todas as cavidades foram encontradas acidentalmente no exame radiográfico completo, no qual apenas filmes intrabucais foram utilizados. Em seguida, foram realizadas radiografias extrabucais apenas nos pacientes em que as radiografias periapicais não possibilitaram a visualização dos limites das cavidades. Os exames radiográficos demonstraram que a maioria das cavidades estava confinada à porção esponjosa do osso, além de apresentar a borda interior da mandíbula interrompida, de maneira que, à palpação, uma reentrância poderia ser percebida. ${ }^{1}$

A depressão óssea citada pode se apresentar com outras nomenclaturas, tais como: cisto ósseo de Stafne, depressão mandibular lingual de glândula salivar, cisto ósseo latente, cisto ósseo estático e defeito da cortical lingual mandibular. ${ }^{2}$

Cirurgiã-dentista, Faculdade Unime de Ciências Agrárias e da Saúde, Lauro de Freitas, BA, Brasil.

Graduanda em Odontologia, Faculdade Unime de Ciências Agrárias e da Saúde, Lauro de Freitas, BA, Brasil.

Graduando em Odontologia, Faculdade Unime de Ciências Agrárias e da Saúde, Lauro de Freitas, BA, Brasil.

Mestre em Cirurgia e Traumatologia Bucomaxilofacial, Universidade de Pernambuco. Doutor em Estomatologia, Universidade Federal da Paraíba. Professor titular da Universidade Estadual de Feira de Santana. Professor do Curso de Odontologia da Faculdade Unime de Ciências Agrárias e da Saúde, Lauro de Freitas, BA, Brasil.

Especialista em Estomatologia pela Faculdade Unime de Ciências Agrárias e da Saúde. Mestre em Estomatologia Clínica, Pontifícia Universidade Católica do Rio Grande do Sul. Professora do Curso de Odontologia da Faculdade Unime de Ciências Agrarias e da Saúde e da Uninassau, Pituba e Lauro de Freitas, BA, Brasil.

-**** Doutora em Processos Interativos dos Órgãos e Sistemas, Instituto de Ciências da Saúde, Universidade Federal da Bahia. Professora do Curso de Odontologia da Faculdade Unime de Ciências Agrárias e da Saúde, Lauro de Freitas, BA, Brasil. 
Esta condição é primeiramente detectada em radiografias, apresentando-se por imagem radiolúcida, unilocular, bem circunscrita, geralmente localizada abaixo do canal mandibular, podendo ser unilateral ou bilateral na porção posterior da mandíbula ou, menos frequentemente, na região anterior da mandíbula. Por ser uma variação anatômica, o DOS não apresenta sintomatologia. ${ }^{3}$

De acordo com a literatura, o DOS tem etiologia incerta, havendo controvérsias quanto a natureza, origem e conteúdo. Contudo, existe a hipótese de que uma pressão exercida na cortical do osso mandibular pela glândula salivar forme a concavidade; outra hipótese é de que, durante o desenvolvimento, uma porção da glândula fica aprisionada no córtex mandibular, podendo também haver o envolvimento de tecidos linfoide e vascular, de gordura e de músculo. ${ }^{4-9}$ Devido à falta de relatos na literatura em pacientes jovens, Shimizu et al. ${ }^{7}$ (2006) sugere não válida a hipótese de origem congênita.

Sobre a epidemiologia, a literatura relata maior incidência em homens; a maioria dos casos relatados é em pessoas de meia idade e em idosos, sendo crianças raramente afetadas. ${ }^{6,7}$

O diagnóstico do DOS se dá por exames radiográficos de rotina, uma vez que as características da alteração não fornecem dados suficientes para detecção clínica da sua presença. A radiografia panorâmica é geralmente o primeiro exame a revelar imagens do defeito ósseo. ${ }^{9}$

O diagnóstico diferencial da cavidade de Stafne deve ser realizado com uma ampla variedade de lesões, tais como: cisto ósseo solitário, cisto ósseo traumático, cisto apical, displasia fibrosa, ameloblastoma, tumor odontogênico queratocístico. ${ }^{10} \mathrm{Os}$ exames imaginológicos são indispensáveis ao diagnóstico diferencial, pois fornecem informações precisas quanto às condições dos tecidos moles e duros em questão sem que haja a necessidade de expor o paciente a procedimentos cirúrgicos exploratórios. ${ }^{6}$ Como a radiografia panorâmica fornece uma imagem bidimensional, há a necessidade de realizar um exame que forneça uma imagem mais detalhada, tridimensional: a tomografia computadorizada (TC). ${ }^{1}$

Os primeiros trabalhos utilizando TC foram realizados pelo engenheiro Hounsfield na década de 1970 e resultaram no prêmio Nobel de Medicina, em 1979. A TC é um exame de aquisição volumétrica, ou seja, permite obter imagens tridimensionais, eliminando, dessa maneira, a sobreposição de estruturas anatômicas, bem como tem a capacidade de diferenciar tecidos moles e estruturas ósseas. ${ }^{11}$

De acordo com o envolvimento com as corticais e o osso medular e com a profundidade do defeito, o DOS é classificado em subtipos: I) concavidade limitada à cortical lingual; II) concavidade da cortical lingual que envolve os espaços medulares; III) depressão da cortical lingual, envolvendo osso medular, e abaulamento da cortical vestibular; IV) continuidade/perfuração da cortical vestibular. ${ }^{6}$
Assim, os objetivos do presente trabalho foram apresentar um relato de caso de paciente diagnosticado com DOS e ressaltar a importância da TC na confirmação do diagnóstico.

\section{Relato de caso}

Paciente do sexo masculino, faioderma, 74 anos de idade, buscou atendimento na Clínica do Idoso da União Metropolitana de Cultura e Educação (Unime). A queixa principal do paciente quando chegou à unidade era "o dente quebrou". Na história atual da doença, foi relatada uma fratura em um dente anterior superior, em que não foi mencionada sintomatologia dolorosa. Foram realizados exames clínicos e físicos, e, após a avaliação de rotina, foi solicitada a radiografia panorâmica.

Foi visualizada na radiografia panorâmica uma área radiolúcida, unilocular, circunscrita, localizada abaixo do canal mandibular, em corpo mandibular esquerdo, estendendo-se para região basilar da mandíbula, sugestiva de DOS (Figura 1).

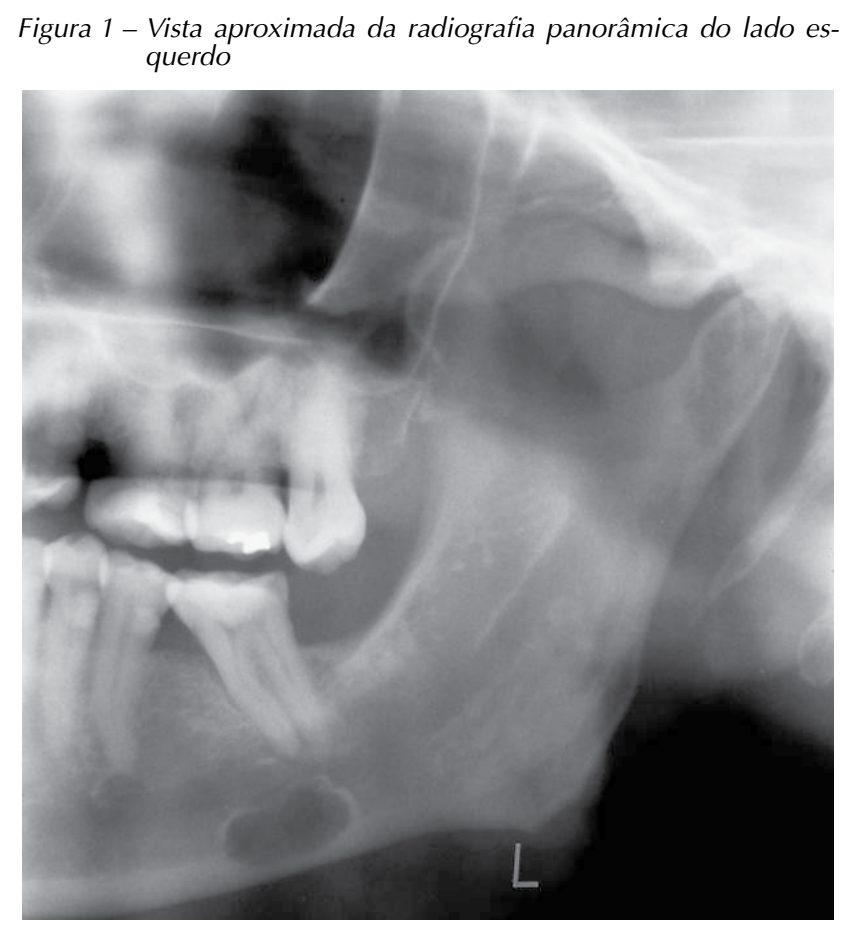

Fonte: autores.

Em seguida, foi solicitado exame de TC fan beam (TCFB), que apresentou imagem hipodensa, de contornos precisos e regulares, de limites hiperatenuantes, localizados no corpo mandibular à esquerda, inferiormente ao canal mandibular, sem envolvimento deste ou da cortical óssea adjacente, medindo $1,5 \mathrm{~cm}$ por $0,8 \mathrm{~cm}$ nos maiores eixos ( $\mathrm{Fi}$ gura 2). Nos cortes sagitais e axiais, identificou-se presença de depressão óssea na região lingual, confirmando a integralidade do corpo mandibular e a hipótese de diagnóstico de DOS (Figura 3A e 3B). 
Figura 2 - Tomografia computadorizada da mandíbula (corte sagital)

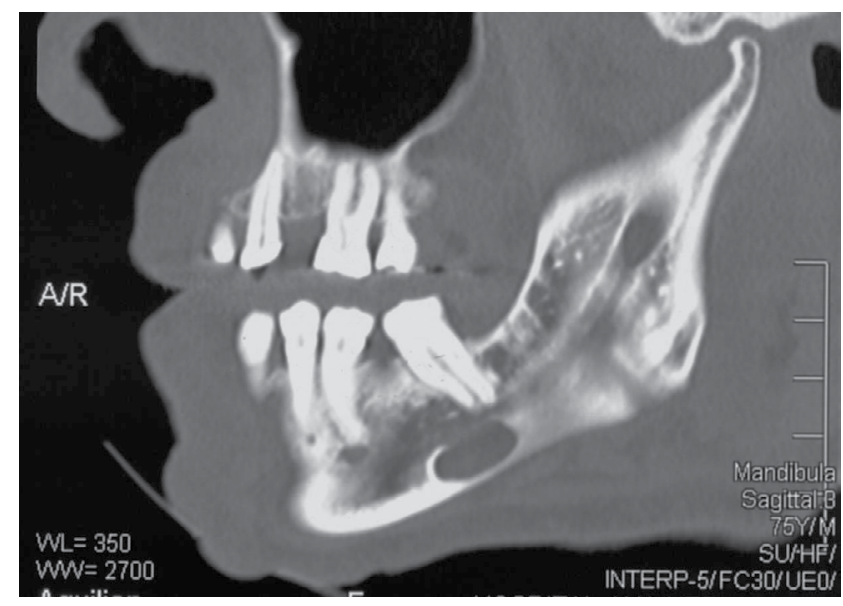

Fonte: autores.

Figura 3 - Tomografia computadorizada da mandíbula: A) corte coronal; B) corte axial.
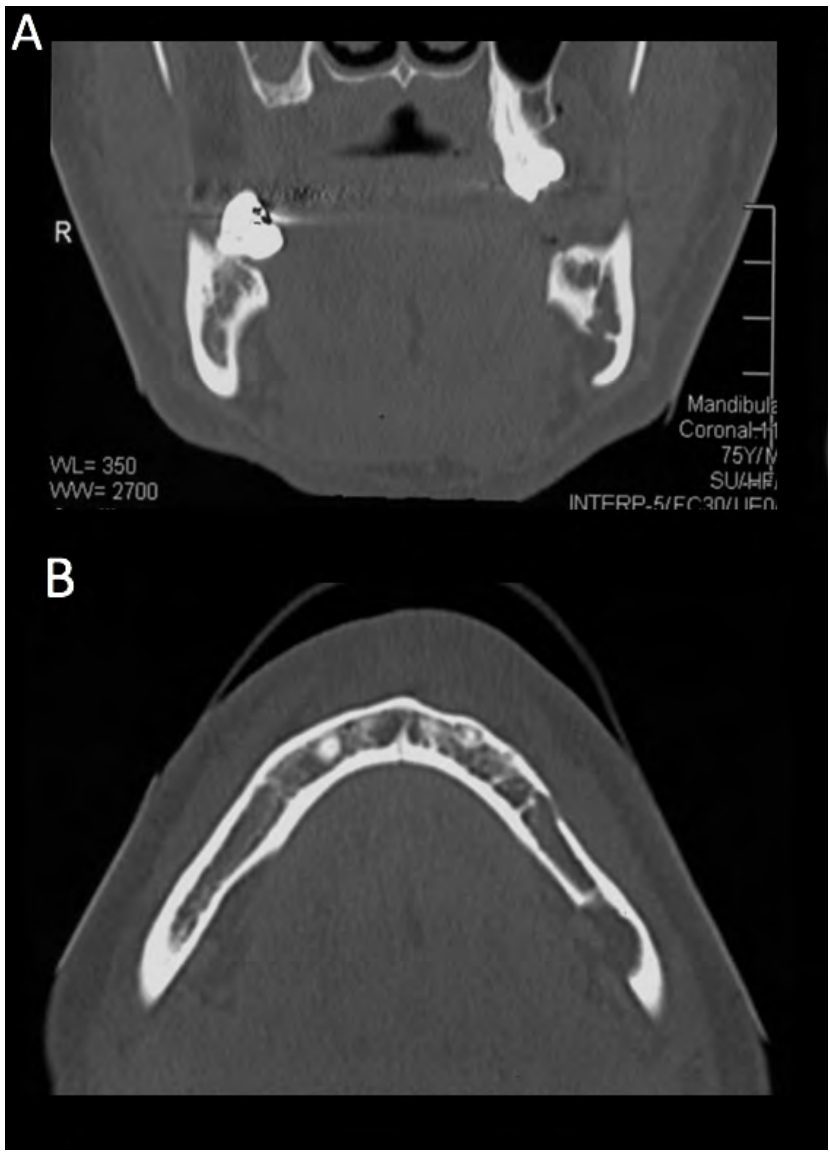

Fonte: autores.

Diante das características clínicas e dos exames imaginológicos, a conduta de escolha foi a proservação do caso, devido à confirmação do DOS. Após um ano e meio, foi solicitada nova radiografia panorâmica (Figura 4), em que foi evidenciada a imagem com características radiográficas e dimensões semelhantes à primeira radiografia. $\mathrm{O}$ paciente aceitou a publicação do presente caso clínico por meio de assinatura de um termo de consentimento livre e esclarecido.
Figura 4 - Radiografia panorâmica um ano e meio após o diagnóstico

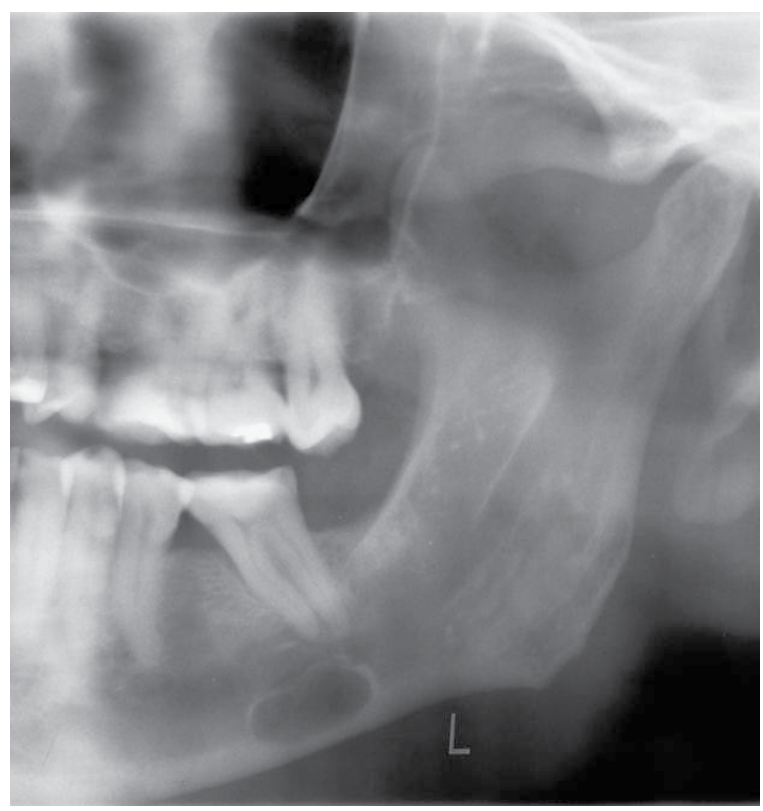

Fonte: autores.

\section{Discussão}

Neste caso clínico, a hipótese de DOS foi observada primeiramente em uma radiografia panorâmica de rotina, corroborando com outros autores., ${ }^{3,4,9,12}$ Este exame imaginológico bidimensional apresenta sobreposição de estruturas anatômicas e não permite a visualização da profundidade da imagem, ${ }^{13}$ não sendo apropriado para o diagnóstico definitivo.

A solicitação da TC para a confirmação de diagnóstico desta variação anatômica concorda com a literatura pesquisada, pois as informações contidas na literatura afirmam que a avaliação com TC geralmente é suficiente para o diagnóstico final, sendo utilizada como método de diagnóstico não invasivo. ${ }^{4,9,12,14}$ No caso relatado, as imagens obtidas por TC evidenciaram uma lesão sem envolvimento do canal mandibular ou cortical ósseo, sendo compatível com o subtipo II, de acordo com as classificações de Ariji. ${ }^{6}$

A TC com janela para tecido mole, a sialografia ou um procedimento invasivo avalia a presença de tecido glandular no interior da lesão, ${ }^{4}$ além da ressonância magnética, que pode ser necessária para um diagnóstico definitivo. ${ }^{9}$ A biópsia é indicada nos casos atípicos ou na presença de suspeita de lesões patológicas. ${ }^{3}$ Como o paciente deste relato não apresentou alterações atípicas relacionadas à imagem, apenas a TC foi suficiente para o diagnóstico de DOS.

Comparando a TCFB e a TC cone beam na avaliação de lesões patológicas das regiões de cabeça e pescoço, observa-se que existem custo reduzido e benefício elevado para o paciente, quando é solicitada a TC cone beam. As doses de radiação são quinze vezes menores; o custo financeiro, mais baixo; as imagens têm alta qualidade de detalhes; e pouco ar- 
tefato é produzido na presença de metais. ${ }^{8,15}$ Contudo, o paciente deste relato realizou a TCFB, devido ao fato de o exame ser disponibilizado pelo Sistema Único de Saúde.

Diante dos achados clínicos e imaginológicos, foi adotada a conduta de proservação do caso, com a realização de radiografias panorâmicas periódicas, para avaliação de possíveis alterações de tamanho, o que condiz com a literatura estudada. ${ }^{3,4}$ Assim, com a utilização da TC, procedimentos invasivos podem ser evitados, principalmente em pacientes idosos, quando os exames imaginológicos sugerem como diagnóstico o DOS. ${ }^{12}$

\section{Considerações finais}

A TCFB apresentou uma imagem côncava na parede lingual da mandíbula, descartando a possibilidade de um processo patológico e confirmando a hipótese de diagnóstico de DOS.

A utilização da TC como ferramenta complementar aos achados clínicos e radiográficos evitou submeter o paciente idoso a procedimentos invasivos desnecessários para a construção do diagnóstico definitivo. Assim, por se tratar de uma variação anatômica, a conduta foi a proservação do caso.

\section{Abstract}

Objective: the objective of the following work was reported in the case of an elderly male patient. Clinical case: male patient, 74 years-old, who was attended at the Dentistry Clinic of the Metropolitan Union of Education and Culture, and after a panoramic radiograph, an image suggestive of the defect was evidenced. patient oriented to perform a Fan Beam Computed Tomography scan for diagnostic confirmation. Final considerations: the Stafne bone defect is defined as a lingual depression in the mandible, rounded or ovoid, ranging from 1 to 3 $\mathrm{cm}$ in diameter, with very dense and well defined bone margins. It presents, radiographically, as a radiolucent, circumscribed and unilocular image in the posterior region of the mandible, below the mandibular canal. It is usually detected in routine radiographic examinations as a panoramic radiograph, being a confirmation of the diagnosis obtained by performing the Fan Beam Computed Tomography or Cone Beam, due to obtaining images that allow a three-dimensional evaluation, thus discarding other types of injuries. Because it is an anatomical variation, the conduct is to preserve the case.

Keywords: Bone cysts. Diagnosis. Mandible. Tomography.

\section{Referências}

1. Villoria EM, Cunha CHRD, Francio LA, Noia J, Taitson P, Spyrides KS, et al. Diagnóstico do defeito ósseo de Stafne por meio de tomografia computadorizada de feixe cônico: relato de caso. Facul de Odontol de Lins/Unime 2013; 23(1):53-8.
2. Arantes EBR, Lopes TS, Valentim BBP, Curvelo JAR, Guedes, FR, Janini MER. Defeito ósseo de Stafne em região anterior: relato de caso. Rev Bras Odontol, Rio de Janeiro 2017; 74(1):77-9.

3. Münevveroglu AP, Aydin KC. Stafne Bone Defect: Report of two Cases. Hidawi Pub Corp 2012; Art ID 654839:1-5.

4. Nikzad S, Azari A, Khezri FH. Diagnosis of a Lingual Mandibular Bone Defect (Stafne's Bone Defect) by CT. Scan Iran J Radiol 2010; 7(1):27-30.

5. Araújo F, Marques T, Correia A, Silva A, Tinoco J. Differential diagnosis of a salivary gland bone defect by means of computerized tomography: A case report. Revodontcienc 2009; 24(2):218-20.

6. Ávila LD, Dias-Ribeiro E, Sampieri MBS, Ferreira-Júnior O, Santa'ana E. Cisto ósseo de Stafne - área radiolúcida na mandíbula versus patologias ósseas: revisão de literatura. Rev Cir Traumatol Buco Maxilofac Camaragibe 2009; 9(3):35-42.

7. Shimizu M, Osa N, Okamura K, Yoshiura K. CT analysis of the Stafne's bone defects of the mandible. Dentomaxillofac Radiol 2006; 35:95-102.

8. Caputo BV, Felicori SM, Giovani EM, Costa C. Validação da tomografia computadorizada de feixe cônico (CBCT) como recurso no diagnóstico do defeito ósseo lingual na mandíbula. Rev Inst Ciênc Saúde 2009; 27(4):422-5.

9. Sisman Y, Eto OA, Mavili E, Sahman H, Ertas ET. Anterior Stafne bone defect mimicking a residual cyst: a case report. Dentomaxilofac Radiol 2010; 39(2):124-6.

10. Pozuelo A, Assis M, López-Quiles J, Pozuelo E. Cavidad idiopática de Stafne. A propósito de um caso clínico. Cient Dent 2007; 4(3):217-21.

11. Cavalcanti MGP, Sales MAO. Tomografia computadorizada. In: Cavalcanti MGP. Diagnóstico por Imagem da Face. São Paulo: Livraria Santos; 2008. p. 3-43.

12. Ogunsalu C, Pillai K, Barclay S. Radiological Assessment of Type II Stafne Idiopathic Bone Cyst in a Patient Undergoing Implant Therapy: A Case Report. West Indian Med J 2006; 55(6):447-50.

13. Caputo BV, Felicori SM, Giovani EM, Costa C. Validação da tomografia computadorizada de feixe cônico (CBCT) como recurso no diagnóstico do defeito ósseo lingual na mandíbula. Rev Inst Ciênc Saúde 2009; 27(4):422-5.

14. Abuabara A, Baratto Filho F, Cruz GV, Guerino L, Giovanini A. Cone beam tomography evaluation of lingual cortical mandibular defect diagnosed as Stafne boné cavity. Rev Sul-Bras Odont 2009; 6:104-6.

15. Garib DG, Raymundo Jr R, Raymundo MV, Raymundo DV, Ferreira SN. Tomografia computadorizada de feixe cônico (Cone beam): entendendo este novo método de diagnóstico por imagem com promissora aplicabilidade na Ortodontia. Rev Dental Press Ortop Facial 2007; 12(2):139-56.

\section{Endereço para correspondência:}

Juliana Andrade Cardoso

Av. Praia de Itapoan, quadra 19, lote 6, SMF Villas Trade, loja 5, Vilas do Atlântico

42700-000, Lauro de Freitas, Bahia, Brasil

Telefone: (71) 99108-6718

E-mail: juliandradec@gmail.com

Recebido: 04/10/18. Aceito: 22/10/18. 